\title{
Level and timing of physical activity during normal daily life in depressed and non-depressed individuals
}

Olga Minaeva $\mathbb{Q}^{1}$, Sanne H. Booij $\mathbb{0}^{2,3}$, Femke Lamers ${ }^{4}$, Niki Antypa ${ }^{5}$, Robert A. Schoevers ${ }^{1}$, Marieke Wichers ${ }^{1}$ and Harriëtte Riese $^{1}$

\begin{abstract}
Engaging in physical activity is known to reduce depressive symptoms. However, little is known which behavioral factors are relevant, and how patterns of activity change during depressive episodes. We expected that compared to controls, in depressed individuals the level of activity would be lower, the amplitude of 24-h-actigraphy profiles more dampened and daytime activities would start later. We used 14-day continuous-actigraphy data from participants in the Netherlands Study of Depression and Anxiety (NESDA) who participated in an ambulatory assessment study. Participants with a depression diagnosis in the past 6 months $(n=58)$ or its subsample with acute depression (DSM diagnosis in the past 1 month, $n=43)$ were compared to controls without diagnoses $(n=63)$. Depression was diagnosed with a diagnostic interview. Actigraphy-derived variables were activity mean levels (MESOR), the difference between peak and mean level (amplitude) and the timing of the activity peak (acrophase), which were estimated with cosinor analysis. Compared to the control group, both depression groups (total: $B=-0.003, p=0.033$; acute: $B=$ $-0.004, p=0.005)$ had lower levels of physical activity. Amplitude was also dampened, but in the acute depression group only (total: $B=-0.002, p=0.065$; acute: $B=-0.003, p=0.011$ ). Similarly, the timing of activity was marginally significant towards a later timing of activity in the acute, but not total depression group (total: $B=0.206, p=0.398$; acute: $B=0.405, p=0.084$ ). In conclusion, our findings may be relevant for understanding how different aspects of activity (level and timing) contribute to depression. Further prospective research is needed to disentangle the direction of the association between depression and daily rest-activity rhythms.
\end{abstract}

\section{Introduction}

Large epidemiological studies indicate that physical activity (PA) is negatively associated with the prevalence of depressive disorders and severity of depressive symptoms ${ }^{1-3}$, and that exercise can improve depressive symptoms in nonclinical ${ }^{4,5}$ and clinical populations ${ }^{6,7}$. Besides effects of altered levels of PA on depression, changes in diurnal rhythm activity patterns, which include

\footnotetext{
Correspondence: Olga Minaeva (o.minaeva@umcg.nl)

${ }^{1}$ Interdisciplinary Center for Psychopathology and Emotion Regulation,

Department of Psychiatry, University Medical Center Groningen, University of Groningen, Groningen, The Netherlands

${ }^{2}$ Department of Developmental Psychology, Faculty of Behavioural and Social Sciences, University of Groningen, Groningen, The Netherlands

Full list of author information is available at the end of the article
}

chronobiological factors, may be involved. Unraveling the different aspects of PA that tap into depression may be helpful for further understanding of the pathogenesis of depression and for future practical implementation. Therefore, we will examine these two possibilities using unique data from 2-week continuous actigraphy in both depressed and healthy individuals.

Prior studies examined the association between PA levels and depressive symptoms and disorders. The effect is mostly explained as that more PA generates positive emotions $^{8}$, or has a favorable, neurotrophic impact on the brain $^{9}$. In line with this, lower levels of activity were found to predispose to affective depressive symptoms, such as depressed mood and anhedonia, which are the core 
symptoms of depressive disorders ${ }^{10}$. Also, healthy individuals with low self-reported PA were at risk for developing depression, while higher PA levels were protective against developing depression among older adults ${ }^{11}$ and a general population ${ }^{12}$. Both studies using self-report measures of the amount of PA, as well as studies with objective actigraphy measures, confirmed the negative association between activity and depression ${ }^{13-18}$.

While prior actigraphy studies provided valuable insights, they have several limitations. These studies were conducted in small samples (40-54 participants) ${ }^{13,15,16}$, mostly used relatively short assessment periods (i.e. between $12 \mathrm{~h}$ and 7 days) ${ }^{13,15,17}$, and examined specific subgroups of patients ${ }^{14,17}$, with the notable exception of the study of Difrancesco et al. ${ }^{18}$. The latter study conducted 14 days of actigraphy measurements in a large sample of participants $(n=359)$. However, more than 7 days of measurement are needed to obtain reliable estimates of PA for most variables of interest ${ }^{19}$. A recent study reported a reduced overall level of PA among individuals with either anxiety or depression episode in the last 6 months compared to remitted and healthy controls $^{18}$. A substantive point in how this current study is different from previous ones, however, is the investigation of the timing of activity together with the level of activity.

Physical activity may be associated with depression due to changes in diurnal activity rhythm. The circadian rhythm, which regulates various physical, mental, and behavioral changes during a $24-\mathrm{h}$ cycle $^{20}$, can be advanced or delayed in depressed individuals with regards to the timing of activity ${ }^{21}$ or dampened ${ }^{13}$. This may cause shifting in the preferred timing of PA in depressed individuals $^{22,23}$, possibly expressed as sleep problems, changed sleep duration ${ }^{24}$ or less exposure to beneficial daylight ${ }^{25}$. Therefore, the timing of PA may be an important factor in the association between PA and depression. Further support for this assumption comes from studies on chronotype that report the association between evening type and more depressive symptoms ${ }^{26-29}$ or depression ${ }^{30-32}$.

Among actigraphy studies on the timing of daily PA, one showed that the later timing of PA is associated with increased risk of developing depressive symptoms among elderly men ${ }^{14}$. Two other studies, in elderly women ${ }^{33}$ and in a small group of outpatients ${ }^{13}$, did not find a significant association with the timing of PA, but only a tendency towards a later timing among depressed individuals. Although studies mentioned above have provided insight in the timing of activity, their findings are inconclusive and mostly focused on specific age and population groups $^{14,33}$.

Currently, it is unclear whether associations between PA and depression originate only from a lower level of $\mathrm{PA}$, or whether this association additionally arises from shifted timing of PA due to a change in diurnal rhythm. The latter was even less studied using actigraphy measurements in depressed populations. As it is important to know what behavioral factors are at play in order to optimize targets for intervention, we will study both the level and timing of activity. The current study also complements previous studies by simultaneously using objective measures of both the level and timing of PA, measured by continuous actigraphy over 2 weeks. We use partly the same data as Difrancesco et al. ${ }^{18}$, but focus specifically on individuals with depressive disorders, excluding individuals with pure anxiety disorders. Additionally, we will focus on individuals with an acute diagnosis (within the past month), on top of individuals with diagnosis of depression in the past 6 months. Studying a more homogeneous (i.e. acutely), depressed group allows us to examine acute symptoms and not merely residual symptoms during recovery from latter depressive episodes.

We also use a fine-grained methodology, which allows us to look at diurnal activity patterns with a longer assessment period than most previous studies and in both depressed and healthy individuals. In the current study, we hypothesized (1) that depressed individuals have lower levels of PA than controls, (2) depressed individuals have dampened diurnal rhythms compared to healthy controls, and (3) become active later during the day due to a shift in their circadian phase.

\section{Materials and methods \\ Participants}

We used data from the Netherlands Study of Depression and Anxiety (NESDA, www.nesda.nl). Details about NESDA were given extensively before ${ }^{34}$. In short, NESDA is an ongoing multisite naturalistic longitudinal cohort study among 2981 adults (18-65 years) at baseline, including individuals with depressive disorders and/or anxiety disorders, as well as healthy controls, which were recruited from community, primary care, and specialized mental health-care settings. Patients with a psychotic disorder, bipolar disorder, obsessive-compulsive disorder, or substance use disorder, and persons not fluent in Dutch were excluded from the baseline assessment. The NESDA study was approved by the VUmc ethical committee (reference number 2003/183) and all respondents gave informed consent.

In the current study, we used a subsample $(n=121)$ from wave 6 (9-year follow-up), which contained a 14-day period of ecological momentary assessments. Details of the actigraphy measurements are given elsewhere ${ }^{18}$. Of the 384 initially included participants, 14 had no available actigraphy data for several reasons, such as technical failure, 10 individuals had less than 1 weekday or weekend-day data available. For this study, we examined 
the effect of a depressive episode; therefore, participants with remitted depressive disorder $(n=152)$, with anxiety disorder only $(n=67)$ and the siblings of NESDA participants $(n=20)$ were excluded. As comorbidity of anxiety and depressive disorders is common ${ }^{35}$, this was not an exclusion criterion. Actigraphy data from individuals with an episode of depressive disorder (major depressive disorder and/or dysthymia) in the past 6 months (called "total depression group", $n=58$ ) and from individuals with no lifetime depressive and anxiety disorder (controls, $n=63$ ) were used. Additionally, we created a subgroup of the total depression group consisting of people with a depressive episode in the past month (called "acute depression group", $n=43$ ).

\section{Actigraphy}

Participants completed 14 consecutive days of all-day actigraphy activity monitoring. They wore a GENEActiv actigraphy device on a nondominant wrist. Participants were instructed to wear the watch day and night and only taking it off when going to the sauna or when playing a contact sport in which wearing a wristband is unsafe. The device is a triaxial wrist accelerometer recording data in SI units represented as acceleration in three axes $(x, y$, and $z$ ). GENEActiv validity studies have demonstrated strong correlations for criterion validity (Pearson $r=$ $0.79-0.98)^{36}$ and a good ability to determine sedentary behavior in adults (18-55 years) (Pearson $r=0.81)^{37}$. To pre-process the raw data, an open-source $\mathrm{R}$ package, GGIR (version 1.5-12) ${ }^{38}$ was used. At the first stage of pre-processing, we verified sensor calibration error using local gravity as a reference, checked for abnormally high values, non-wear periods, and then extracted objective PA measures. During raw data cleaning, missing data (e.g. suspected of monitor non-wear) or invalid data were imputed by the averages at similar time points on different days of the week, as GGIR does by default. In this way, within-day variability of the data should not be affected, unlike the situation when the data are imputed with the average of close by time points of the same day. Only respondents with valid actigraphy data for at least $16 \mathrm{~h}$ per day, and at least 10 days were included in the analysis $(n=121)$. To estimate PA, we calculated a metric ENMO (Euclidean Norm Minus One) using the formula $\sqrt{x^{2}+y^{2}+z^{2}}-1 g$ with any negative values rounded up to zero and by reducing such measure over 1-min epochs $^{39}$. We adapted and executed the R-script for the current study.

\section{Predictor and outcome measures}

The main predictor in the study was clinical group status (depression diagnosis versus healthy control status). Diagnoses were acquired with the Composite International Diagnostic Interview (CIDI, version 2.1) based on the DSM-IV ${ }^{40}$. The CIDI has high interrater reliability, high test-retest reliability, and high validity for depressive disorders ${ }^{40}$. Participants were divided into the following groups based on the results of a CIDI assessment: (1) a group with no lifetime history of psychiatric disorders (healthy controls) $(n=63)$, and (2) a group with a depressive disorder (either with or without anxiety comorbidity in the past 6 months, $n=58$ ). In a next step, to assess whether there was an acute state effect of depression on PA, we selected a subgroup of the 6-month depressed group with a depressive disorder present in the past 1 month (termed "acute", $n=43$ ) in the analysis to compare to the control group.

The main outcome variables were three actigraphyderived PA variables: mean levels of activity (MESOR, an acronym for Medline Estimated Statistic of Rhythm), height of peak with respect to the mean level (amplitude), and the timing of the activity peak (acrophase). MESOR, as a measure of the mean level of the curve between the highest and the lowest point, represents the average activity level of the day. Amplitude, as a measure of the difference between activity during the day and the night, indicates the robustness of the diurnal rhythm. Acrophase, as a measure of the time of the day when the activity peak occurred, indicates timing preferences of an individual during the day. MESOR and amplitude were measures for the level of PA, and acrophase-a measure for the timing of PA. To calculate these variables, circadian rhythms were estimated by fitting individual 1-min epoch ENMO data to a cosine curve of a 24-h activity rhythm, which was obtained by the cosinor method ${ }^{41,42}$ using the following equation: $y(t)=M+A \cos (2 \pi t / \tau+\phi)$ $+e(t)$, where $y$ is the selected variable, $t$ is the elapsed time, $M$ is MESOR, a rhythm-adjusted mean, $A$ is the amplitude of circadian rhythm, $\tau$ is the period of the circadian rhythm (the 24-h period), $\phi$ is the acrophase, and $e(t)$ is the error term.

Cosinor analysis was performed in R statistical software version $1.1 .383^{43}$. For this purpose, we created an R script, which obtains daily values (i.e. 14 values per person) of the cosinor parameters MESOR, amplitude, and acrophase, and automates this process across persons and days and exporting the values to a new data set. The script is given in Supplementary Materials 1.

\section{Chronotype}

Chronotype was assessed with the Munich Chronotype Questionnaire (MCTQ) ${ }^{44}$. The MCTQ is not a scale; therefore, its reliability cannot be assessed. The MCTQ highly correlates with the Morningness-Eveningness Questionnaire (MEQ) $(r=-0.73)^{45}$, which in turn, consistently report high levels of reliability $(>0.80)^{46}$. Chronotype was defined as the midpoint in time between falling asleep and waking up on free days corrected for 
"oversleep" due to the sleep debt that individuals accumulate over the workweek (MSFsc).

\section{Potential confounding variables}

Demographics (gender, age, marital status), socioeconomic status (education, employment) ${ }^{12}$, smoking status, $\mathrm{BMI}^{47}$, current medication use (e.g. benzodiazepine, antidepressants $)^{13}$, and depression severity were included in the analysis as additional covariates. Depression severity was assessed with the Inventory of Depressive Symptomatology (IDS) ${ }^{48}$. Internal consistency of the IDS-SR is high, and the Cronbach $\alpha$ ranged from 0.67 to $0.94^{48,49}$. Current medication use was based on drug container inspection and medications were coded according to the World Health Organization Anatomical Therapeutic Chemical (ATC) classification and considered present if participants reported currently using psychopharmacological medication.

\section{Statistical analysis}

Baseline characteristics were compared pairwise between the control group and the total depression (diagnosis in the past 6 months) or acute depression (diagnosis in the past 1 month) group, respectively. The independent $t$ test was used for continuous variables (age, BMI, education, IDS scores) and Chi-squared test was used for categorical variables (gender, marital status, employment, smoking status, IDS severity groups, and psychopharmaca use).

As the data are hierarchically structured, we used multilevel linear modeling to assess whether the MESOR, amplitude, and acrophase of the PA rhythm differed between individuals with and without the diagnosis of depression. A two-level data structure was used where days of actigraphy were defined as the first-level unit and individuals as the second-level unit. The first set of analyses was performed using the 6-month cut-off for an episode of depressive disorder (total depression group). Two models were run for each outcome variable. The first model assessed the association between group (nondepressed $=0$, depressed $=1$ ) and PA levels and timing (Model 1). Next, this association was tested while including all covariates (Model 2). Only for acrophase, a third model (Model 3) was tested as an additional check whether chronotype (also an indicator of the phase of the circadian rhythm) explains (part of) the association between acrophase and group status. A significant association would support the hypothesis that a shift in acrophase indeed reflects a shift in the circadian rhythm.

For the second set of analyses, all models for three outcome variables (MESOR, amplitude, and acrophase) were repeated in the subsample of acutely depressed (1month cut-off, $n=43$ ) versus controls.

Besides the fixed effects (group status and the covariates), a random intercept and time trend (day number) were included if they improved the model fit. Model fit was assessed using the Akaike Information Criterion (AIC) (lower is better). For all models, a random intercept, but not a slope for day number, improved the model fit. The best fitting covariance structure for the random effects was the variance components (VC) structure. In addition, to correct for potential autocorrelation of the residuals, which is often present in repeated assessments data, we examined several possible covariance structures for the residuals. The best structure was an autoregressive heterogeneous $(\mathrm{AR}(1) \mathrm{H})$. Multilevel analyses were conducted using SPSS Statistics version 25. Significance levels were set at $p<0.05$.

The residuals of the final models were not normally distributed. To overcome this issue, we repeated all analyses with a bootstrap approach, as bootstrapping is robust against violations of normality ${ }^{50}$. Due to model complexity, we needed to slightly adapt the model; instead of specifying an $\mathrm{AR}(1) \mathrm{H}$ covariance structure for the residuals, we included a lag(1) outcome variable as a fixed and random predictor. This way, potential autocorrelation was still addressed. Even though the bootstrap analysis gave similar results, and in three cases borderline significant findings even became significant, we decided to keep a more conservative approach to prevent overstating the results. Therefore, we reported the results of the original models only. However, the syntax and the results of the bootstrap analyses are given in the Supplementary Materials 2-4 (Tables S1 and S2).

\section{Results \\ Sample description}

Demographic and clinical characteristics of the participants are given in Table 1. Participants $(n=121)$ have a mean age of $52.13(\mathrm{SD}=11.3$; range $28-72)$ years, and $63.6 \%$ were women. No significant differences in age, gender, marital status, BMI, and chronotype were found between the total depression group and controls. Depressed individuals were more often unemployed and smokers, had lower education, higher mean depressive symptom scores, and used more medication compared to the control group. The comparisons of the acute depression $(n=43)$ group to the control group gave similar results. Mean values for circadian rest-activity characteristics (MESOR, amplitude, and acrophase) across groups are given in Table 2. As expected, effect sizes are somewhat larger in the 1-month depression 
Table 1 Demographic and clinical characteristics of depressed patients and controls $(n=121)$.

\begin{tabular}{|c|c|c|c|c|c|}
\hline & \multirow{2}{*}{$\begin{array}{l}\text { Total depression group } \\
\text { (6 months) } \\
(n=58)\end{array}$} & \multirow{2}{*}{$\begin{array}{l}\text { Acute depression group } \\
(1 \text { month })^{\mathrm{a}} \\
(n=43)\end{array}$} & \multirow{2}{*}{$\begin{array}{l}\text { Control group } \\
(n=63)\end{array}$} & \multicolumn{2}{|l|}{$p$ value } \\
\hline & & & & $6 \mathrm{~m} /$ control $^{b}$ & $1 \mathrm{~m} /$ control $^{\mathrm{c}}$ \\
\hline \multicolumn{6}{|l|}{ Demographics } \\
\hline Age, years: mean $(S D)^{d}$ & $52.34(10.59)$ & $52.14(9.57)$ & $51.94(12.05)$ & 0.844 & 0.927 \\
\hline Gender, female: $n(\%)^{\mathrm{e}}$ & $36(62.10)$ & $29(67.40)$ & $41(65.10)$ & 0.850 & 0.837 \\
\hline Education, years: mean (SD) ${ }^{d}$ & $12.17(3.16)$ & $11.81(3.16)$ & $13.94(2.82)$ & 0.002 & $<0.001$ \\
\hline Marital status, married: $n(\%)^{e}$ & $28(48.30)$ & $22(51.20)$ & $36(57.10)$ & 0.194 & 0.396 \\
\hline Employment, yes: $n(\%)^{\mathrm{e}}$ & $22(38.00)$ & $15(34.90)$ & $48(76.20)$ & $<0.001$ & $<0.001$ \\
\hline \multicolumn{6}{|l|}{ Lifestyle } \\
\hline Smoking status, yes: $n(\%)^{e}$ & $20(34.50)$ & $17(39.50)$ & $7(11.10)$ & 0.020 & 0.007 \\
\hline Body mass index (BMI): mean (SD) ${ }^{d}$ & $27.36(4.92)$ & $27.48(5.07)$ & $26.06(5.57)$ & 0.178 & 0.181 \\
\hline \multicolumn{6}{|l|}{ Psychiatric disorders } \\
\hline Only depressive disorders: $n(\%)$ & $7(12.10)$ & $1(2.30)$ & $0(0)$ & - & - \\
\hline $\begin{array}{l}\text { Depressive and anxiety disorders: } \\
\text { n (\%) }\end{array}$ & $51(87.90)$ & $42(97.70)$ & $0(0)$ & - & - \\
\hline \multicolumn{6}{|l|}{ Psychological scales } \\
\hline IDS, raw score: mean (SD) ${ }^{\mathrm{d}, \mathrm{f}}$ & $30.34(13.10)$ & $34.53(11.73)$ & $5.02(3.00)$ & $<0.001$ & $<0.001$ \\
\hline \multicolumn{6}{|l|}{ IDS, severity: $n(\%)^{\mathrm{e}}$} \\
\hline None & $8(13.80)$ & $3(7.00)$ & $63(100)$ & $<0.001$ & $<0.001$ \\
\hline Mild & $11(19.00)$ & $4(9.30)$ & - & & \\
\hline Moderate & $25(43.10)$ & $22(51.20)$ & - & & \\
\hline Severe & $9(15.50)$ & $9(20.90)$ & - & & \\
\hline Very severe & $5(8.60)$ & $5(11.60)$ & - & & \\
\hline \multicolumn{6}{|l|}{ Chronotype } \\
\hline MSFsc, h: mean (SD) $)^{\mathrm{d}, \mathrm{g}}$ & $3.63(1.70)$ & $3.60(1.71)$ & $3.61(1.35)$ & 0.956 & 0.945 \\
\hline \multicolumn{6}{|l|}{ Current medication use } \\
\hline Psychopharmaca users: $n(\%)^{e}$ & $30(51.70)$ & $23(53.50)$ & $3(4.80)$ & $<0.001$ & $<0.001$ \\
\hline Antidepressants: $n(\%)$ & $27(46.60)$ & $20(86.90)$ & $0(0)$ & & \\
\hline Benzodiazepines: $n$ (\%) & $7(12.10)$ & $6(26.10)$ & $1(1.60)$ & & \\
\hline Antipsychotics: $n$ (\%) & $4(6.90)$ & $4(17.40)$ & $0(0)$ & & \\
\hline Lithium: $n$ (\%) & $2(3.40)$ & $1(4.30)$ & $0(0)$ & & \\
\hline Other types ${ }^{\text {h: }} n(\%)$ & $0(0)$ & $0(0)$ & $2(3.20)$ & & \\
\hline
\end{tabular}

IDS Inventory of Depressive Symptomatology, 6m 6-month depression group, $1 m$ 1-month depression group, MSFsc mid-sleep time on free days corrected for sleep debt on workdays.

${ }^{a}$ The subgroup of the total depression group.

${ }^{\mathrm{b}} p$ values for the comparison between the total depression group (6 months) and control group.

${ }^{c} p$ values for the comparison between the acute depression group (1 month) and control group.

Independent $t$ test.

${ }^{\text {e}}$ Chi-squared test (two-sided).

fDepression Severity: 0-13-None, 14-25-Mild, 26-38-Moderate, 39-48-Severe, 49-84-Very severe.

${ }^{9}$ Chronotype differences were not significant and therefore interpreted as negligible across the groups.

${ }^{\mathrm{h}}$ Anticonvulsants and smoking cessation medication.

$P$-values at or below 0.05 are marked in bold. 
Table 2 Mean values for MESOR, acrophase and amplitude on a person level across groups $(n=121)$.

\begin{tabular}{|c|c|c|c|c|c|}
\hline \multirow[t]{2}{*}{ Characteristic } & \multirow[t]{2}{*}{ Total depression group } & \multirow[t]{2}{*}{ Acute depression group ${ }^{a}$} & \multirow[t]{2}{*}{ Control group } & \multicolumn{2}{|c|}{ Cohen's $d$} \\
\hline & & & & $1 / 0^{b}$ & $2 / 0^{b}$ \\
\hline MESOR, mean (SD) & $0.024(0.007)$ & $0.023(0.007)$ & $0.027(0.006)$ & 0.500 & 0.657 \\
\hline Amplitude, mean (SD) & $0.020(0.007)$ & $0.019(0.006)$ & $0.022(0.006)$ & 0.357 & 0.503 \\
\hline Acrophase, mean (SD) & $14.757(1.122)$ & $14.860(1.180)$ & $14.604(0.918)$ & 0.150 & 0.244 \\
\hline
\end{tabular}

${ }^{a}$ The subgroup of the total depression group.

b $1 / 0$ - total depression group compared to controls; 2/0-acute depression group compared to controls.

group than in the 6-month depression group regarding all three outcome variables.

\section{Actigraphy differences between depressed participants (6- month cut-off) and controls}

Having a diagnosis of depressive disorder in the past 6 months was significantly associated with lower MESOR in both the unadjusted (Model 1) and adjusted model (Model 2). For amplitude, group status was significant in the unadjusted model (Model 1) and was associated with a lower amplitude which can be interpreted as a dampened rhythm, but not in the adjusted model (Table 3, Model 2, $p=0.065)$. Group status was not significantly associated with acrophase expressed in any model, but there was a significant relationship between chronotype and acrophase with later chronotype having later acrophase (Model 3). To ease interpretation of the statistical results, averaged 24-h activity rhythms are visualized per group in Fig. 1.

\section{Actigraphy differences between acutely depressed participants (1-month cut-off) and controls}

Table 4 shows the results of the repeated analyses in a subsample of depressed individuals who were more likely to suffer from acute depressive symptoms. Acute depressive disorders were significantly associated with decreased MESOR in both unadjusted and adjusted models. Acute depressive disorders were associated with significantly lower amplitude in both unadjusted and adjusted models, which indicates the dampened rhythm in the depressed group. Acute depressive disorders were not significantly associated with acrophase in any model, but there was a marginally significant effect towards the later timing of activity in the adjusted model. There was a significant relationship between chronotype and acrophase with later chronotype having later acrophase. The subgroup analysis in this more homogeneous depressed group revealed overall more pronounced results.

\section{Discussion}

We assessed objective actigraphy-measured level and timing of PA in depressed individuals and controls.
Supporting the first hypothesis, MESOR, the rhythmcorrected mean level, was lower in depressed individuals, compared to controls. This result was confirmed in both total depression (6-month) and in the subset of acute depression (1-month) group. The amplitude, as a measure of the difference between activity during the day and the night, was also lower in the subgroup of acutely depressed individuals compared to controls, but not in the total depression group. Our hypothesis that depressed individuals showed a later timing of PA during their normal daily life was not confirmed, notwithstanding the marginally significant association towards the later timing in the acute depression group.

The finding that average PA levels were significantly reduced in depressed individuals compared to the control group is consistent with findings from other actigraphy studies $^{13-15,18}$, which used slightly different approaches for estimating average 24-h activity levels. Interestingly, for the level, we do find effects in both the total and the acute depression groups, suggesting that lower levels of activity may also be present during a recovery phase of a depressive episode. Lower activity could, therefore, be interpreted as a relative persistent vulnerability marker for depression and may even be present before developing depression or relapsing into a next episode. This interpretation, however, is speculative and needs to be confirmed in future prospective research.

Another important finding with respect to the diurnal rhythm of activity is that dampened amplitude was found in the acute depression group. Although these results could either mean that depressed individuals reach lower activity levels at daytime or that they have more nighttime activity than controls, activity pattern (Fig. 1) illustrates that the difference in amplitude results from the first possibility. As the association was only statistically significant among acutely depressed individuals compared to controls, but not in the larger group of individuals who were not all necessarily in an active depressive episode (total depression group), this suggests that dampened amplitude is a state effect of depression. A previous NESDA study showed that the median duration of a depressive episode is 6 months in the sample ${ }^{51}$. Therefore, 


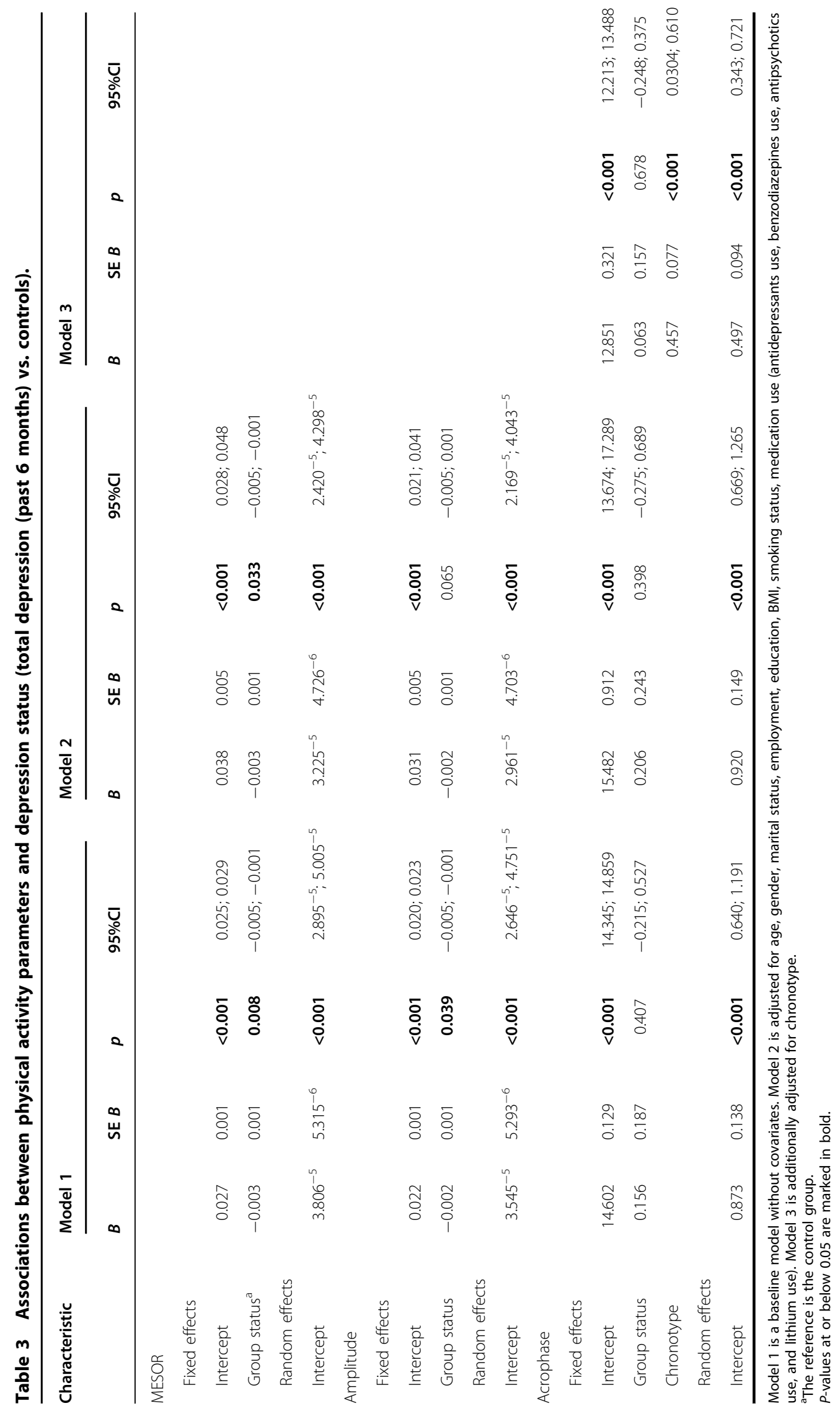




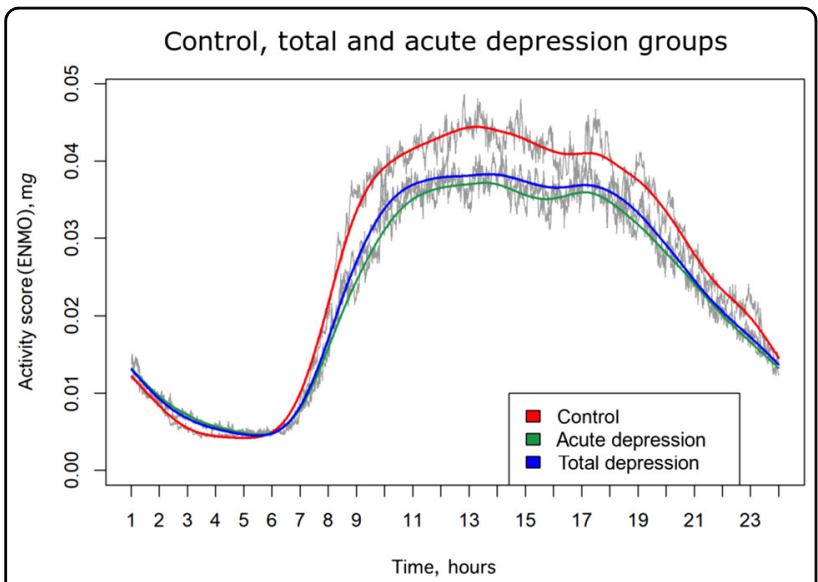

Fig. 1 Twenty-four-hour averaged activity scores across 14 days within different diagnostic (sub-)groups. The acute depression group is the subgroup of the total depression group.

it is possible that some individuals who scored positive on the 6-month threshold had already recovered at the moment of the actigraphy measures. This is supported by the reported IDS scores among the total depression group, which indicates that a third of the subjects had no (13.8\%) or mild depressive symptoms (19\%). Hence, the finding may reflect that the amplitude normalizes and becomes less dampened after individuals recover from their acute depressive episode. Indeed, in line with our findings, studies comparing depressed individuals before and after treatment showed an improvement in intensity and increase in amplitude of daytime activity ${ }^{15}$. In future studies, it may be interesting to explore whether changes in the amplitude do not only go together with a change in depression status but perhaps even precede it. In that case, it could be used as an early-warning signal for onset and recovery.

We found a marginally significant effect towards later timing to be associated with acute depression only in the adjusted model. This nonsignificant finding might be a reflection of an insufficient number of acutely depressed individuals for detecting a relatively small difference in the timing of activity. Additionally, even in the acute depression group, we cannot be sure that everyone was in a depressed state at the moment of assessment. The IDS scores (Table 1) support this idea, as there were some individuals with no (7\%) and mild depressive symptoms $(9.3 \%)$ in the acute depression group also. However, in two prior studies, similar marginally significant effects towards the later timing of activity in depressed individuals compared to controls were reported as well ${ }^{13,33}$. One study had a considerably small sample size $(n=$ $40)^{13}$, and another one used narrow population groups (elderly females); therefore, the findings may not be applicable to other populations ${ }^{33}$. When studying the sleep-wake and activity cycles among young adults with various emerging mental disorders, depressed individuals showed a significantly later acrophase compared to controls $^{52}$. This might be explained by studying much younger participants (20.0 \pm 4.4 years) accompanied by greater medication use and comorbidities (that presume more severe depressive symptoms) and/or more eveningness. Similarly, a later peak of 24-h activity was observed in young ( $\leq 39$ years, 45 min of delay) compared to older participants ( $\geq 40$ years, $30 \mathrm{~min}$ of delay), and in those with more depressive symptoms ${ }^{53}$.

Our study has strengths and limitations. Among the strengths is the large sample size including individuals with clinically diagnosed depressive disorder and individuals without a lifetime history of depression. Moreover, we used the cosinor method, which is one of the most commonly used reliable methods, which allowed us to estimate different characteristics of the level (MESOR and amplitude) and occurrence of the activity peak (acrophase $)^{41,42}$. A limitation of using the cosinor analysis is that it is a parametric analysis, which fits a symmetric cosinor curve into the circadian rhythm. However, the circadian rhythm of daily activity is not symmetric, with a shorter "night-curve" and a longer "day-curve". Moreover, it is known that based on the cosinor method, it is hard to undoubtfully detect when the physically active period starts and how long it lasts. Other nonparametric approaches are available now, but most of them aggregate data across all available days and not day-by-day ${ }^{18}$, reducing power. To overcome this limitation in future studies, more advanced analytical tools have to be used that can improve our understanding of the circadian restactivity rhythm. We also excluded a part of the sample, the remitted depressed individuals, what consequently reduces the sample size. The main reason was that the aim of this study was not originally to include the remitted individuals, as we wanted to compare current cases versus controls. Additionally, it has been recently shown that remitted depressed individuals did not differ from non-depressed in their diurnal rest-activity rhythm ${ }^{18}$. Another limitation of this study is that a small number of individuals in the depression groups reported no or mild depressive symptoms. This was most likely due to logistic reasons typical for large cohort studies as our study is. Participants were diagnosed with the CIDI instrument during the regular NESDA interview wave, which was maximal 31 days prior to the actigraphy assessments, whereas depression severity assessment with the IDS questionnaire was not necessarily done close to the actigraphy assessment period (up to 72 days prior and two cases of 74 and 351 days after the CIDI). Finally, the cross-sectional nature of the study does not allow drawing conclusions regarding the direction of the association between the diagnosis of depression and PA. 


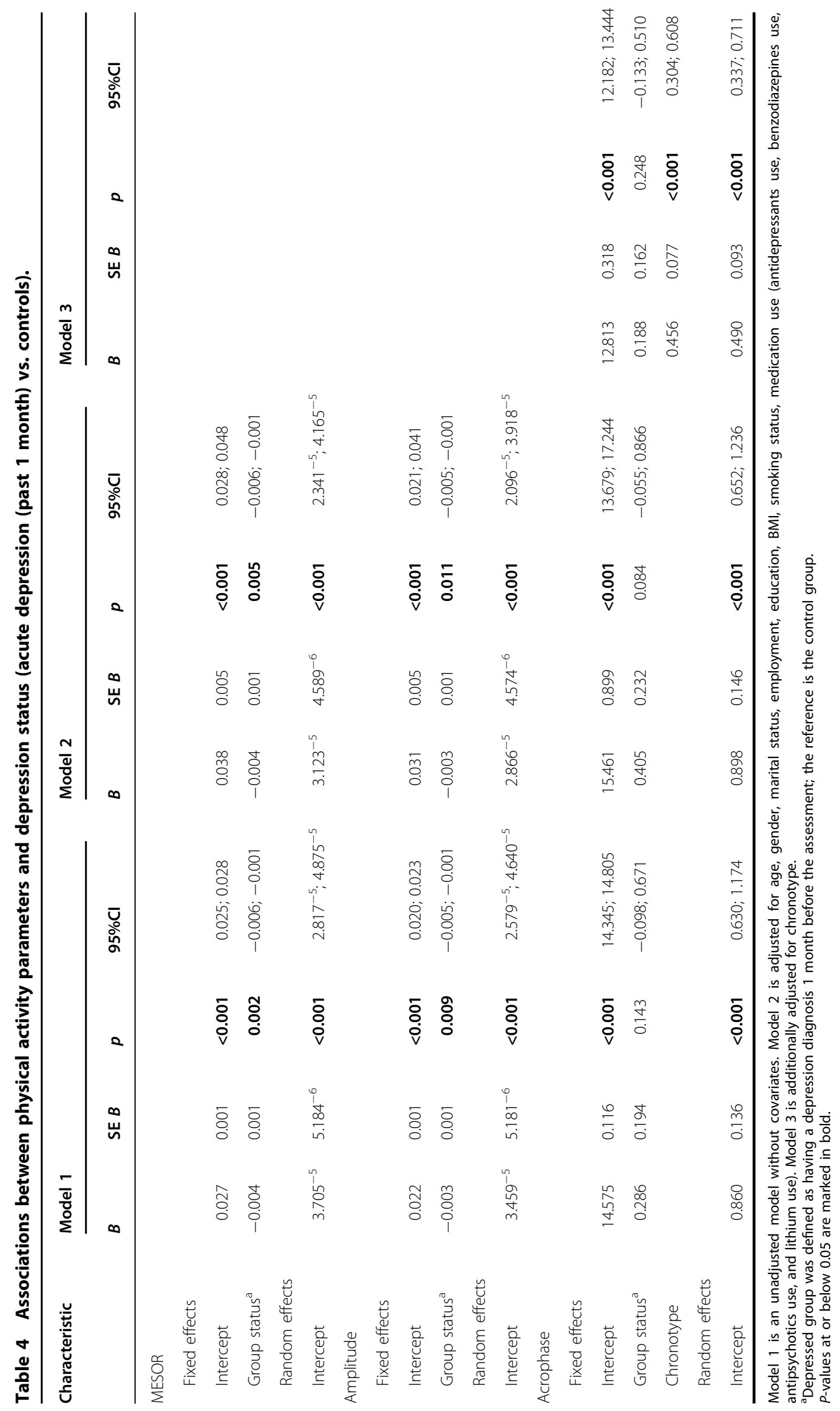


We would recommend for future actigraphy studies in depressed patients to not only study the level and the timing of PA but also rhythm characteristics and their combinations. Eight subgroups with various rhythm characteristics in terms of level, robustness, length, and timing were identified in elderly men ${ }^{14}$. Smagula et al. concluded that different combinations of those characteristics have different associations with depressive symptoms. It might be valuable to use a combination of the cosinor and nonparametric analyses, that will allow taking into account both parametric and nonparametric rhythm-related parameters. Finally, longitudinal studies are needed to disentangle the direction of the association between depression and daily rest-activity rhythms. This knowledge may help to determine a pathophysiologic pathway leading to depressive disorders and to develop better treatments.

In conclusion, our study showed that the level of activity, specifically during the daytime, was significantly lower in individuals in both the total and the acute depression groups compared to controls. Significantly lower day/night differences were found only in the acute depression group. The later timing of activity was only marginally significantly associated with acute depression. These findings not only indicate an important role of the level of PA across both depression groups but also the additional changes in the robustness of the diurnal rhythm (more dampened amplitude of the rhythm), which are associated with the more acute depression state. Hence, our study provided new relevant insights on how different aspects of physical activity, namely level and timing, may be independently associated to depression. Future studies should examine the timing of activity in a larger sample with acutely depressed individuals to be able to draw more firm conclusions.

\section{Acknowledgements}

The infrastructure for the NESDA study (www.nesda.nl) is funded through the Geestkracht program of the Netherlands Organization for Health Research and Development (ZonMw, grant number 10-000-1002) and financial contributions by participating universities and mental health-care organizations (VU University Medical Center, GGZ inGeest, Leiden University Medical Center, Leiden University, GGZ Rivierduinen, University Medical Center Groningen, University of Groningen, Lentis, GGZ Friesland, GGZ Drenthe, Rob Giel Onderzoekscentrum). The authors thank all NESDA participants and research assistants who made the data acquisition possible. We would like to thank Dr. S. de Vos whose statistical expertise was priceless during the data analysis and statistical computations. M. Wichers was supported by funding from the European Research Council (ERC) under the European Union's Horizon 2020 research and innovation program (ERC-CoG-2015; No 681466). M.W. was supported by funding from the European Research Council (ERC) under the European Union's Horizon 2020 research and innovation program (ERC-CoG2015; No 681466).

\footnotetext{
Author details

'Interdisciplinary Center for Psychopathology and Emotion Regulation, Department of Psychiatry, University Medical Center Groningen, University of Groningen, Groningen, The Netherlands. ${ }^{2}$ Department of Developmental Psychology, Faculty of Behavioural and Social Sciences, University of Groningen, Groningen, The Netherlands. ${ }^{3}$ Center for Integrative Psychiatry,
}

Lentis, Groningen, The Netherlands. ${ }^{4}$ Department of Psychiatry, Amsterdam Public Health Research Institute, Amsterdam UMC, Vrije Universiteit, Amsterdam, The Netherlands. ${ }^{5}$ Department of Clinical Psychology, Institute of Psychology, Leiden University, Leiden, The Netherlands

\section{Author contributions}

O.M., S.H.B., M.W. and H.R. were involved in the design of the study. O.M. and S. H.B. performed the statistical analysis. O.M. wrote the original draft of the manuscript. All authors critically revised the draft for intellectual content and approved the final version.

\section{Conflict of interest}

The authors declare that they have no conflict of interest.

\section{Publisher's note}

Springer Nature remains neutral with regard to jurisdictional claims in published maps and institutional affiliations.

Supplementary Information accompanies this paper at (https://doi.org/ 10.1038/s41398-020-00952-w).

Received: 15 July 2019 Revised: 19 May 2020 Accepted: 22 May 2020 Published online: 30 July 2020

\section{References}

1. Goodwin, R. D. Association between physical activity and mental disorders among adults in the United States. Prev. Med. 36, 698-703 (2003).

2. Hamer, M., Stamatakis, E. \& Steptoe, A. Dose-response relationship between physical activity and mental health: the Scottish Health Survey. Br. J. Sports Med. 43, 1111-1114 (2009).

3. Saxena, S., Van Ommeren, M., Tang, K. C. \& Armstrong, T. P. Mental health benefits of physical activity. J. Ment. Health 14, 445-451 (2005).

4. Rebar, A. L. et al. A meta-meta-analysis of the effect of physical activity on depression and anxiety in non-clinical adult populations. Health Psychol. Rev. 9, 366-378 (2015).

5. Conn, V. S. Depressive symptom outcomes of physical activity interventions: meta-analysis findings. Ann. Behav. Med. 39, 128-138 (2010).

6. Ströhle, A. Physical activity, exercise, depression, and anxiety disorders. J. Neural Transm. 116, 777 (2009).

7. Rethorst, C. D., Wipfli, B. M. \& Landers, D. M. The antidepressive effects of exercise: a meta-analysis of randomized trials. Sports Med. 39, 491-511 (2009).

8. Wichers, M. et al. A time-lagged momentary assessment study on daily life physical activity and affect. Health Psychol. 31, 135-144 (2012).

9. Huang, T., Larsen, K. T., Ried-Larsen, M., Møller, N. C. \& Andersen, L. B. The effects of physical activity and exercise on brain-derived neurotrophic factor in healthy humans: a review. Scand. J. Med. Sci. Sport. 24, 1-10 (2014).

10. Stavrakakis, N., Jonge, P., Ormel, J. \& Oldehinkel, A. J. Bidirectional prospective associations between physical activity and depressive symptoms. The TRAILS study. J. Adolesc. Health 50, 503-508 (2012).

11. Strawbridge, W. J., Deleger, S., Roberts, R. E. \& Kaplan, G. A. Physical activity reduces the risk of subsequent depression for older adults. Am. J. Epidemiol. 156, 328-334 (2002).

12. Farmer, M. E. et al. Physical activity and depressive symptom: the Nhanes I epidemiologic follow-up study. Am. J. Epidemiol. 128, 1340-1351 (1988).

13. Hori, H. et al. 24-h activity rhythm and sleep in depressed outpatients. J. Psychiatr. Res. 77, 27-34 (2016).

14. Smagula, S. F. et al. Latent activity rhythm disturbance sub-groups and longitudinal change in depression symptoms among older men. Chronobiol. Int. 32, 1427-1437 (2015).

15. Todder, D., Caliskan, S. \& Baune, B. T. Longitudinal changes of day-time and night-time gross motor activity in clinical responders and non-responders of major depression. World J. Biol. Psychiatry 10, 276-284 (2009).

16. Berle, J. O., Hauge, E. R., Oedegaard, K. J., Holsten, F. \& Fasmer, O. B. Actigraphic registration of motor activity reveals a more structured behavioural pattern in schizophrenia than in major depression. BMC Res. Notes 3, 149 (2010).

17. Burton, C. et al. Activity monitoring in patients with depression: a systematic review. J. Affect Disord. 145, 21-28 (2013). 
18. Difrancesco, S. et al. Sleep, circadian rhythm, and physical activity patterns in depressive and anxiety disorders: a 2-week ambulatory assessment study. Depress. Anxiety 36, 975-986 (2019).

19. Aadland, E. \& Ylvisåker, E. Reliability of objectively measured sedentary time and physical activity in adults. PLOS ONE 10, e0133296 (2015).

20. Wirz-Justice, A. Chronobiology and mood disorders. Dialogues Clin. Neurosci. 5, 315-325 (2003).

21. Quera Salva, M. A. et al. Circadian rhythms, melatonin and depression. Curr. Pharm. Des. 17, 1459-1470 (2011).

22. Germain, A. \& Kupfer, D. J. Circadian rhythm disturbances in depression. Hum. Psychopharmacol. 23, 571-585 (2008).

23. Wirz-Justice, A. Biological rhythm disturbances in mood disorders. Int. Clin. Psychopharmacol. 21(Suppl. 1), 11 (2006).

24. van Mill, J. G., Vogelzangs, N., Van Someren, E. J. W., Hoogendijk, W. J. G. \& Penninx, B. W. J. H. Sleep duration, but not insomnia, predicts the 2-year course of depressive and anxiety disorders. J. Clin. Psychiatry 75, 119-126 (2014).

25. Lewy, A. J., Sack, R. L., Stephen Miller, L. \& Hoban, T. M. Antidepressant and circadian phase-shifting effects of light. Science (80-). 235, 352-354 (1987).

26. Kitamura, S. et al. Evening preference is related to the incidence of depressive states independent of sleep-wake conditions. Chronobiol. Int. 27, 1797-1812 (2010).

27. Levandovski, R. et al. Depression scores associate with chronotype and social jetlag in a rural population. Chronobiol. Int. 28, 771-778 (2011).

28. Merikanto, I. et al. Evening types are prone to depression. Chronobiol. Int. $\mathbf{3 0}$ 719-725 (2013).

29. Selvi, Y. et al. Associations between chronotype, sleep quality, suicidality, and depressive symptoms in patients with major depression and healthy controls. Chronobiol. Int. 27, 1813-1828 (2010).

30. Antypa, N., Vogelzangs, N., Meesters, Y., Schoevers, R. \& Penninx, B. W. J. H. Chronotype associations with depression and anxiety disorders in a large cohort study. Depress. Anxiety 33, 75-83 (2016).

31. Knapen, S. E. et al. Social jetlag and depression status: results obtained from the Netherlands Study of Depression and Anxiety. Chronobiol. Int. 35, 1-7 (2018).

32. Merikanto, I. et al. Circadian preference links to depression in general adult population. J. Affect Disord. 188, 143-148 (2015).

33. Maglione, J. E. et al. Depressive symptoms and circadian activity rhythm disturbances in community-dwelling older women. Am. J. Geriatr. Psychiatry 22, 349-361 (2014)

34. Penninx, B. W. J. H. et al. The Netherlands Study of Depression and Anxiety (NESDA): rationale, objectives and methods. Int. J. Methods Psychiatr. Res. 17, 121-140 (2008).

35. Kessler, R. C. et al. National Comorbidity Survey Replication: the epidemiology of Major Depressive Disorder: results from the National Comorbidity Survey Replication (NCS-R). Jama 289, 3095-3105 (2003).

36. Esliger, D. W. et al. Validation of the GENEA accelerometer. Med. Sci. Sports Exerc. 43, 1085-1093 (2011).
37. Pavey, T. G., Gomersall, S. R., Clark, B. K. \& Brown, W. J. The validity of the GENEActiv wrist-worn accelerometer for measuring adult sedentary time in free living. J. Sci. Med Sport. 19, 395-399 (2016).

38. van Hees, V. T. et al. Autocalibration of accelerometer data for free-living physical activity assessment using local gravity and temperature: an evaluation on four continents. J. Appl. Physiol. 117, 738-744 (2014).

39. van Hees, V. T. et al. Separating movement and gravity components in an acceleration signal and implications for the assessment of human daily physical activity. PLOS ONE 8, e61691 (2013).

40. Wittchen, H. U. Reliability and validity studies of the WHO-Composite International Diagnostic Interview (CIDI): a critical review. J. Psychiatr. Res. 28, 57-84 (1994).

41. Cornelissen, G. Cosinor-based rhythmometry. Theor. Biol. Med. Model. 11, 1-24 (2014).

42. Nelson, W. Methods for cosinor-rhythmometry. Chronobiologia 6, 305-323 (1979).

43. R. Core Team. R: A Language and Environment for Statistical Computing ( $R$ Foundation for Statistical Computing, Vienna, Austria, 2017).

44. Roenneberg, T. Epidemiology of the human circadian clock. Sleep. Med. Rev. 11, 429-438 (2007).

45. Zavada, A., Gordijn, M. C. M., Beersma, D. G. M., Daan, S. \& Roenneberg, T. Comparison of the Munich Chronotype Questionnaire with the Horne-Östberg's morningness-eveningness score. Chronobiol. Int. 22, 267-278 (2005)

46. Di Milia, L., Adan, A., Natale, V. \& Randler, C. Reviewing the psychometric properties of contemporary circadian typology measures. Chronobiol. Int. 30 , 1261-1271 (2013)

47. Kaplan, M. S., Newson, J. T., McFarland, B. H. \& Lu, L. Demographic and psychosocial correlates of physical activity in late life. Am. J. Prev. Med. 21, 306-312 (2001).

48. Rush, A. J., Gullion, C. M., Basco, M. R., Jarrett, R. B. \& Trivedi, M. H. The Inventory of Depressive Symptomatology (IDS): psychometric properties. Psychol. Med 26, 477-486 (1996).

49. Cusin, C., Yang, H., Yeung, A. \& Fava, M. Rating scales for depression. In Handbook of clinical rating scales and assessment in psychiatry and mental health. Humana Press, Totowa, NJ, pp 7-35 (2009).

50. Barker, L. E. \& Shaw, K. M. Best (but oft-forgotten) practices: Checking assumptions concerning regression residuals. Am. J. Clin. Nutr. 102, 533-539 (2015).

51. Penninx, B. W. J. H. et al. Two-year course of depressive and anxiety disorders: results from the Netherlands Study of Depression and Anxiety (NESDA). J. Affect Disord. 133, 76-85 (2011).

52. Robillard, R. et al. Ambulatory sleep-wake patterns and variability in young people with emerging mental disorders. J. Psychiatry Neurosci. 40, 28-37 (2015).

53. Robillard, R. et al. Sleep-wake cycle in young and older persons with a lifetime history of mood disorders. PLOS ONE 9, e87763 (2014). 